\title{
Tratamento combinado na dissecção de artéria mesentérica superior: desafio terapêutico
}

\author{
Combination treatment for superior mesenteric artery dissection: therapeutic challenge \\ Guilherme Borgo Ficagna ${ }^{1,2}$ (D), Cristiano do Carmo Galindo ${ }^{1}$ (D), Jean Paulo Niero Mazon ${ }^{3}$ (D), \\ Gustavo Galvan Debiasi',2 (D), Amanda Bogo Vargas² (D), Laura Sahd Bernz² (D)
}

\begin{abstract}
Resumo
A dissecção da artéria mesentérica superior é uma causa rara de dor abdominal, com quadro clínico variável. Seu diagnóstico é difícil, e não existe consenso sobre suas opções terapêuticas; elas variam em torno de tratamento conservador, correção aberta, endovascular ou combinada. Descrevemos o caso de um homem de 45 anos com dissecção isolada da artéria mesentérica superior, com quadro de dor abdominal persistente após tentativa de tratamento conservador. Ele foi submetido à revascularização cirúrgica aberta devido à localização e complexidade da dissecção. O tratamento com endarterectomia, arterioplastia com remendo de pericárdio bovino e acesso retrógrado para abertura da mesentérica com stent foi realizado com sucesso. A angina abdominal foi totalmente resolvida após estabilização do quadro. A combinação de abordagem aberta e endovascular deve ser considerada como terapia para casos de dissecção complexa isolada da artéria mesentérica superior.
\end{abstract}

Palavras-chave: artéria mesentérica superior; dissecção; endarterectomia; angioplastia.

\begin{abstract}
Dissection of the superior mesenteric artery is a rare cause of abdominal pain, with a variable clinical picture. It is difficult to diagnose and there is no consensus on treatment options, which range from conservative treatment to open, endovascular, or combination repair. We describe the case of a 45-year-old man with isolated dissection of the superior mesenteric artery and persistent abdominal pain after conservative treatment had been attempted. He underwent open surgical revascularization due to the location and complexity of the dissection. Treatment consisting of endarterectomy, arterioplasty with bovine pericardium patch, and retrograde access to open the mesenteric artery with a stent was successful. Abdominal angina was completely resolved after the condition had stabilized. A combination of open and endovascular approaches should be considered as treatment for cases of isolated complex dissection of the superior mesenteric artery.
\end{abstract}

Keywords: superior mesenteric artery; dissection; endarterectomy; angioplasty.

Como citar: Ficagna GB, Galindo CC, Mazon JPN, Debiasi, GG, Vargas AB, Bernz LS. Tratamento combinado na dissecção de artéria mesentérica superior: desafio terapêutico. J Vasc Bras. 2022;21:e20210157. https://doi.org/10.1590/16775449.210157

\footnotetext{
${ }^{1}$ Hospital Maternidade Marieta Konder Bornhausen - HMKB, Itajaí, SC, Brasil.

${ }^{2}$ Universidade do Vale do Itajaí - UNIVALI, Itajaí, SC, Brasil.

${ }^{3}$ Hospital de Clínicas de Porto Alegre - HCPA, Porto Alegre, RS, Brasil.

Fonte de financiamento: Nenhuma.

Conflito de interesse: Os autores declararam não haver conflitos de interesse que precisam ser informados.

Submetido em: Agosto 02, 2021. Aceito em: Novembro 22, 2021.
} 


\section{INTRODUÇÃO}

A dissecção da artéria mesentérica superior (AMS) é uma causa rara de dor abdominal e de difícil diagnóstico ${ }^{1,2}$. A tomografia computadorizada protagoniza o método mais reconhecido para a detecção da doença, uma vez que evidencia a lesão na maioria das vezes e auxilia no planejamento da abordagem ${ }^{3,4}$. As opções terapêuticas para a dissecção de AMS são variáveis e vão do tratamento conservador ao reparo cirúrgico aberto ${ }^{5}$, endovascular ou combinado ${ }^{6,7}$, dependendo da gravidade do caso. O projeto foi aprovado pelo Comitê de Ética e Pesquisa (CAAE 47854621.0.0000.0120, parecer consubstanciado 4.875.653).

\section{PARTE I: CASO CLÍNICO}

Paciente masculino de 45 anos, hipertenso e ex-tabagista, com quadro de dor abdominal difusa e recorrente com 30 dias de evolução, com piora no último dia, sem demais queixas associadas. Ele mantinha sinais vitais estáveis e, ao exame físico abdominal, apresentava dor leve à palpação, sem sinais de irritação peritoneal. Foi submetido a tomografia com contraste, que evidenciou dissecção isolada da AMS (Figura 1), mantendo perfusão de todos os seus ramos. Nesse primeiro momento, optou-se por internação hospitalar e controle da dor com sintomáticos e antiagregação. Como houve melhora dos sintomas com analgesia e os exames laboratoriais se mantiveram sem alterações durante toda internação, o paciente recebeu alta após 5 dias para manter tratamento e seguimento ambulatorial. Retornou ao hospital no dia seguinte da alta hospitalar referindo piora da dor, sendo novamente submetido a tomografia com contraste, que não evidenciou alteração em relação ao exame anterior. $\mathrm{O}$ paciente reinternou pelo quadro álgico e recebeu alta após 7 dias de internação, estando clinicamente assintomático,

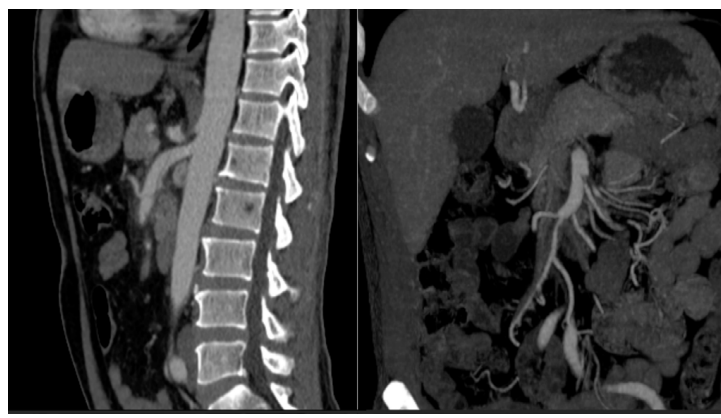

Figura 1. Dissecção isolada de artéria mesentérica superior mantendo perfusão de todos os seus ramos. ainda em uso de antiagregantes. No dia seguinte, retornou novamente ao hospital com quadro de piora da dor, e a imagem de controle evidenciou ausência de fluxo vascular nos ramos distais da AMS (Figura 2), sugerindo tromboembolismo. Nesse momento, o próximo passo seria definir a conduta terapêutica entre as possibilidades de tratamento, sendo elas reparo aberto, endovascular ou combinado.

\section{PARTE II: TERAPÊUTICA}

O paciente foi submetido a arteriografia, porém, devido à complexidade da dissecção e ao risco de oclusão de ramos da AMS, optou-se pela correção cirúrgica aberta. Foi realizada a laparotomia, seguida de endarterectomia do segmento de dissecção da AMS e de arterioplastia com remendo de pericárdio bovino. No primeiro dia de pós-operatório, o paciente evoluiu com piora súbita da dor, e a angiotomografia de controle evidenciou oclusão da AMS na porção distal (Figura 3), sugerindo trombose do enxerto, sendo submetido a nova intervenção com embolectomia de todos os ramos da AMS, além de nova arterioplastia

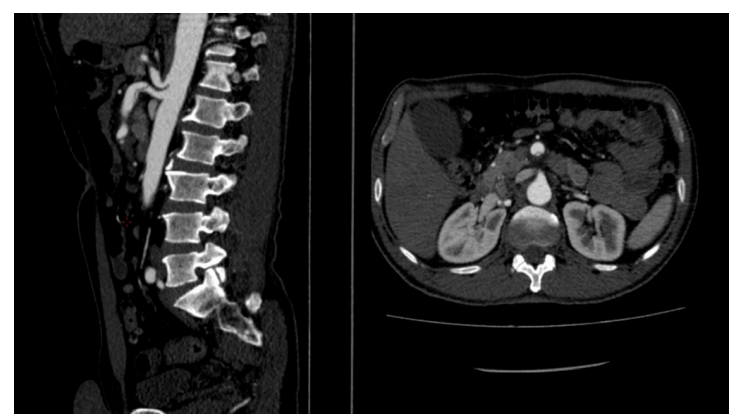

Figura 2. Presença de flap intimal na artéria mesentérica superior sugestivo de dissecção e ausência de fluxo vascular nos ramos distais, sugerindo tromboembolismo.

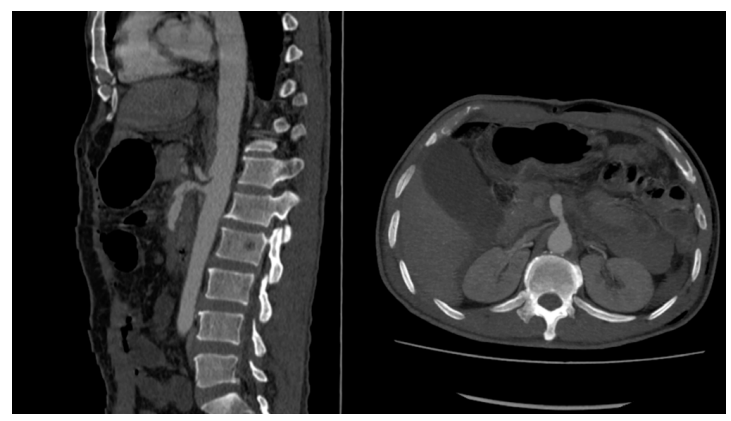

Figura 3. Artéria mesentérica superior pérvia na porção proximal com falha de enchimento abrupta na porção distal, sugerindo trombose do enxerto. 
com extenso remendo de pericárdio (Figura 4). Foi realizado Doppler transoperatório, que evidenciou estenose de $70-80 \%$ na origem da AMS, sendo, então, realizada angioplastia através de punção retrógada do remendo (Figura 5) com implante de stent na origem da AMS (Figura 6) com sucesso e normalização do fluxo (Figura 7) e velocidade ao ultrassom. Observou-se, no

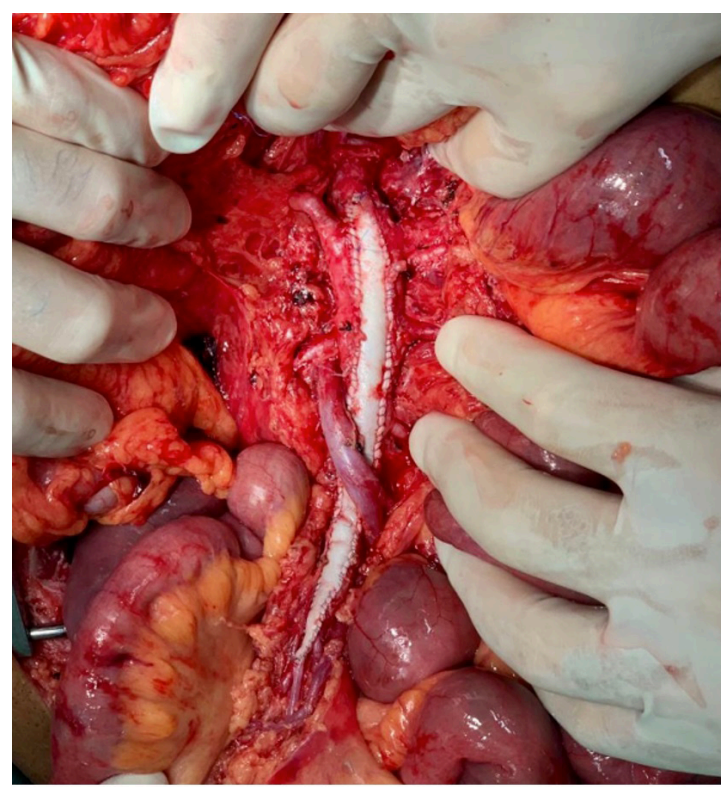

Figura 4. Remendo da artéria mesentérica superior.

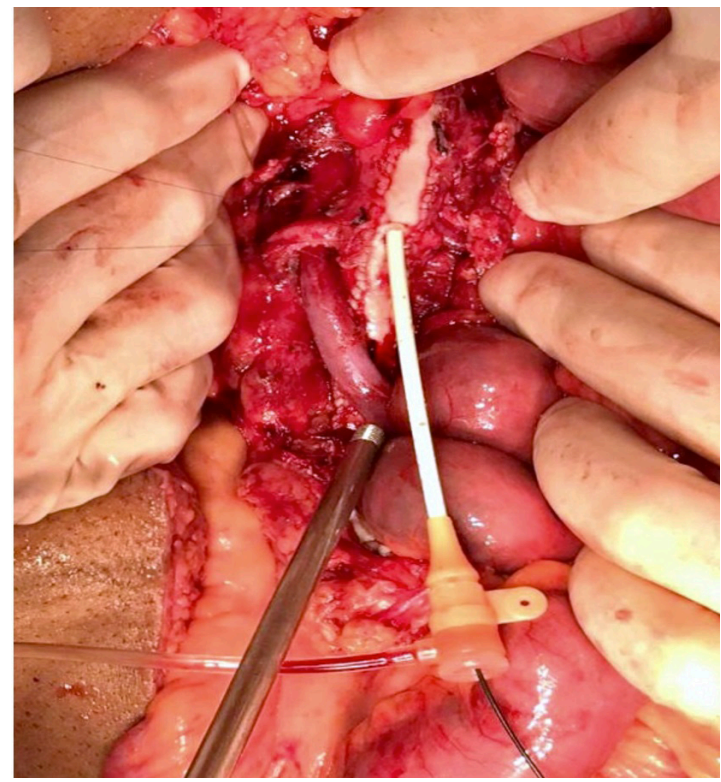

Figura 5. Punção retrógrada do remendo da artéria mesentérica superior. transoperatório, várias áreas de isquemia do segmento intestinal, porém viáveis, sendo programado second look após 24h para revisão. Conforme programado, realizou-se, então, nova abordagem, que evidenciou área de sofrimento de alça, seguida de enterectomia segmentar de aproximadamente $80 \mathrm{~cm}$ de alça de jejuno e enteroanastomose. Após as abordagens, o paciente teve boa evolução na recuperação pós-operatória, apresentando melhora dos sintomas e recebendo alta hospitalar com encaminhamento para seguimento ambulatorial, sendo realizadas consultas mensais e imagens de controle após 3 meses (Figura 8).

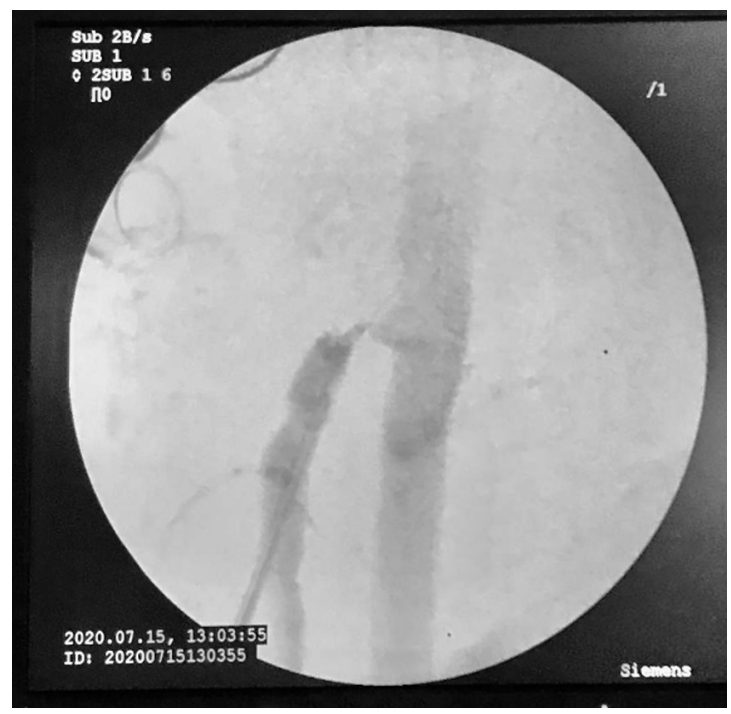

Figura 6. Arteriografia da artéria mesentérica superior.

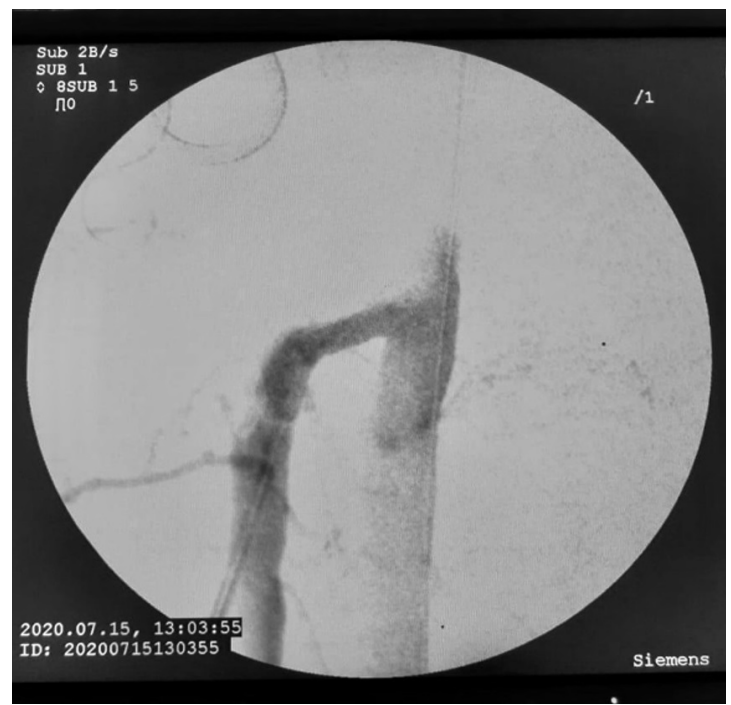

Figura 7. Arteriografia após revascularização da artéria mesentérica superior. 


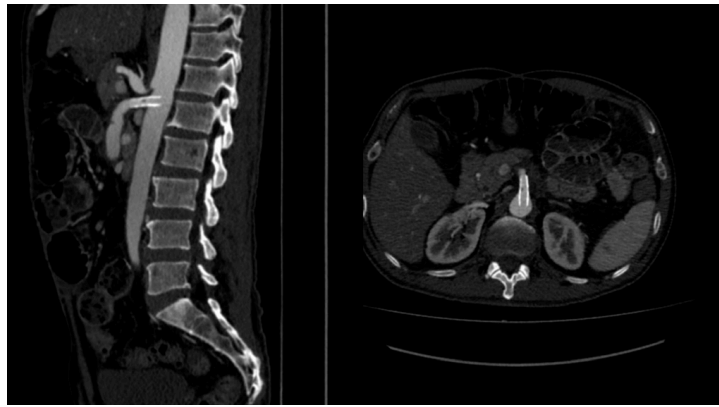

Figura 8. Stent na porção proximal da AMS e sua perviedade.

\section{DISCUSSÃO E CONCLUSÃO}

Apesar da raridade e da etiologia não totalmente esclarecida nos casos não associados a trauma ou iatrogênica, a dissecção da AMS é uma doença grave associada a complicações como formação de trombos, hemorragias e isquemia mesentérica ${ }^{2,8}$. A hipertensão, como no paciente relatado, nem sempre está associada, mas pode ser considerada fator de risco, bem como o tabagismo. A clínica do paciente é variável, podendo a dor abdominal estar presente de forma insidiosa como resultado de isquemia intestinal. Em alguns casos, há presença de náuseas, vômitos, distensão abdominal e até mesmo instabilidade hemodinâmica ${ }^{3,4}$.

A angiografia é o padrão ouro para a decisão do tratamento do paciente, uma vez que ela permite a detalhada avaliação da lesão e de sua gravidade. Há uma diversidade de tratamentos a se seguir - a escolha diverge entre tratamento conservador ${ }^{4}$, endovascular ou cirurgia aberta ${ }^{1,9}$. O tratamento conservador, como foi proposto inicialmente para o caso, é o mais comum, sendo reservado para os casos em que os pacientes se apresentam com estabilidade hemodinâmica e em que não há evidência de ruptura da dissecção da AMS. Esse tratamento é efetivo na maioria dos casos, mantendo-se acompanhamento com exames de imagem e foco nos sinais clínicos de isquemia mesentérica e no suprimento vascular da AMS

As indicações para a cirurgia ainda são controversas e variam de acordo com a apresentação clínica, sinais de complicação e até mesmo recorrência dos sintomas. A laparotomia exploratória está indicada caso o paciente apresente sinais e sintomas de isquemia mesentérica, entretanto as abordagens podem ser complementares, sendo o reparo aberto, endovascular ou combinado $6,10,11$. No nosso caso, a tentativa de tratamento conservador não obteve resolução do quadro, sendo necessária intervenção cirúrgica em que utilizamos ambas as técnicas. A opção entre angioplastia seguida ou não da colocação de stent ${ }^{7,12}$ associada a algum outro método depende de cada situação, demonstrando que a dinâmica para escolha de tratamento depende da apresentação de cada caso e de sua complexidade. Neste caso, o tratamento endovascular foi usado como resgate de uma falha no tratamento aberto inicial, identificada no transoperatório e corrigida com uso de stent. Complicações como isquemia intestinal podem cursar com evolução mesmo após a terapêutica; por isso, sempre é necessária a atenção aos sinais dessa complicação ou, bem como foi optado neste caso, um second look e ressecção intestinal quando houver necessidade.

Como a dissecção de AMS é muitas vezes de difícil diagnóstico ${ }^{13}$ e ainda não existe consenso sobre o melhor tratamento para cada caso ${ }^{8,14}$, os sinais clínicos e o acompanhamento do paciente são essenciais para um desfecho favorável. Quando necessário, deve ser indicada a abordagem cirúrgica mais adequada para cada caso, seja por reparo aberto, endovascular ou combinado.

\section{REFERÊNCIAS}

1. Tanaka S, Fukuda A, Kawakubo E, Matsumoto T. Superior mesenteric artery dissection with prolonged abdominal angina treated by laparotomy, endarterectomy, patch angioplasty, and retrograde open mesenteric stenting: a case report. Surg Case Rep. 2019;5(1):164. http://dx.doi.org/10.1186/s40792-019-0736-0. PMid:31664639.

2. Ullah W, Mukhtar M, Abdullah HM, et al. Diagnosis and management of isolated superior mesenteric artery dissection: A systematic review and meta-analysis. Korean Circ J. 2019;49(5):400-18. http:// dx.doi.org/10.4070/kcj.2018.0429. PMid:31074212.

3. Lee A, Frith K. Letters to the editor. Aust Fam Physician. 2016;106(10):699. PMid:27806458.

4. Yun WS, Kim YW, Park KB, et al. Clinical and angiographic follow-up of spontaneous isolated superior mesenteric artery dissection. Eur J Vasc Endovasc Surg. 2009;37(5):572-7. http://dx.doi.org/10.1016/j. ejvs.2008.12.010. PMid:19208448.

5. Katsura M, Mototake H, Takara H, Matsushima K. Management of spontaneous isolated dissection of the superior mesenteric artery: case report and literature review. World J Emerg Surg. 2011;6:16. http://dx.doi.org/10.1186/1749-7922-6-16. PMid:21549001.

6. Roussel A, Della Schiava N, Coscas R, et al. Results of retrograde open mesenteric stenting for acute thrombotic mesenteric ischemia. J Vasc Surg. 2019;69(4):1137-42. http://dx.doi.org/10.1016/j. jvs.2018.07.058. PMid:30777691.

7. Gao DN, Qi QH, Gong P. Endovascular stenting of spontaneous isolated dissection of the superior mesenteric artery: a case report and literature review. Medicine. 2017;96(46):e8598. http://dx.doi. org/10.1097/MD.0000000000008598. PMid:29145276.

8. Léonard M, Courtois A, Defraigne J-O, Sakalihasan S. Le cas clinique du mois dissection isolée de l'artère mésentérique supérieure. Rev Med Liege. 2017;72(4):175-180.

9. Hoek J, Helleman J, Jansen J. Spontane dissectie van de A. mesenterica superior. Ned Tijdschr Geneeskd. 2016;160(20)

10. Mitsuoka $H$, Nakai $M$, Terai $Y$, et al. Retrograde stent placement for symptomatic spontaneous isolated dissection of the superior 
mesenteric artery. Ann Vasc Surg. 2016;35:203.e17-21. http:// dx.doi.org/10.1016/j.avsg.2016.01.029. PMid:27236096.

11. Tsuji $Y$, Hino $Y$, Sugimoto $K$, Matsuda $H$, Okita Y. Surgical intervention for isolated dissecting aneurysm of the superior mesenteric artery - a case report. Vasc Endovascular Surg. 2004;38(5):469-72. http:// dx.doi.org/10.1177/153857440403800513. PMid:15490047.

12. Casella IB, Bosch MA, Sousa WO Jr. Isolated spontaneous dissection of the superior mesenteric artery treated by percutaneous stent placement: case report. J Vasc Surg. 2008;47(1):197-200. http:// dx.doi.org/10.1016/j.jvs.2007.07.051. PMid:18178474.

13. He Q, Yu F, Fu Y, et al. Evaluation of isolated abdominal visceral artery dissection with multi-scale spiral computed tomography: a retrospective case series. J Cardiothorac Surg. 2021;16(1):61. http://dx.doi.org/10.1186/s13019-021-01428-8. PMid:33781323.

14. Garrett HE Jr. Options for treatment of spontaneous mesenteric artery dissection. J Vasc Surg. 2014;59(5):1433-1439.e1-2. http:// dx.doi.org/10.1016/j.jvs.2014.01.040. PMid:24655752.
Correspondência Guilherme Borgo Ficagna Hospital Maternidade Marieta Konder Bornhausen - HMKB Rua 900, 135, apartamento 602 - Centro 88330-604 - Balneário Camboriú (SC), Brasil Tel.: (47) 99608-9797 E-mail: guilherme.borgo@icloud.com

Informações sobre os autores GBF - Graduado em Medicina, Universidade do Vale do Itajaí (UNIVALI); Residente em Cirurgia Geral, Hospital e Maternidade Marieta Konder Bornhausen (HMMKB).

CCG - Graduado em Medicina, Fundação Universidade Regional de Blumenau (FURB); Residência Médica em Cirurgia Geral, Hospital e Maternidade Marieta Konder Bornhausen (HMMKB); Residência

Médica em Radiologia Intervencionista, Centro de Radiologia Intervencionista e Vascular (CRIVA); Residência Médica em Cirurgia

Vascular e Endovascular, Hospital Real e Benemérita Associação Portuguesa de Beneficência (HBP).

JPNM - Graduado em Medicina; Residência em Cirurgia Geral, Universidade Federal de Santa Maria (UFSM); Residência Médica em Cirurgia Vascular pelo Hospital São Vicente de Paulo (HSVP), Residência Médica em Cirurgia Endovascular, Hospital de Clínicas de Porto Alegre (HCPA).

GGD - Graduado em Medicina, Universidade do Vale do Itajaí (UNIVALI); Residência Médica em Cirurgia Geral, Hospital e Maternidade Marieta Konder Bornhausen (HMMKB); Residência Médica em Cancerologia Cirúrgica, Hospital A. C. Camargo (HACC). ABV e LSB - Acadêmicas de Medicina, Universidade do Vale do Itajaí (UNIVALI).

Contribuição dos autores Concepção e desenho do estudo: GBF Análise e interpretação dos dados: GBF, CCG Coleta de dados: GBF, ABS, LSB

Redação do artigo: GBF, CCG Revisão crítica do texto: JPNM, GGB Aprovação final do artigo*: GBF, CCG, JPNM, GGD, ABS, LSB Análise estatística: N/A Responsabilidade geral pelo estudo: GBF

*Todos os autores leram e aprovaram a versão final submetida ao J Vasc Bras. 